nacci series $0,1,1,2,3,5,8,13, \cdots$ giving the values of the Lucas function $U_{n}$ associated with the polynomial $x^{2}-x-1$. This polynomial is irreducible modulo 13 , so that the period of the Fibonacci series modulo 13 gives the period of the mark $\alpha$ associated with $x^{2}-x-1$ in the finite field of order $13^{2}$. We have $\omega=7$, norm $\alpha=-1, \theta=2, k=2, \sigma=2, p-1=12$. Hence (2) becomes $(2,2)|\delta|(2,12)$, so that $\delta=2$. Hence the period is 28 , which is easily verified directly. It seems quite difficult to determine the exact value of $\delta$ in all cases. ${ }^{*}$

California Institute of Technology

\title{
ON A PROBLEM OF KNASTER AND ZARANKIEWICZ $\dagger$
}

BY J. H. ROBERTS

Knaster and Zarankiewicz have proposed the following problem: $\ddagger$ "Does every continuum $A$ contain a subcontinuum $B$ such that $A-B$ is connected?" Knaster has shown, $\S$ by an example in 3 -space, that the answer is in the negative. In the present paper an example is given of a plane continuum $M$ such that every non-degenerate proper subcontinuum of $M$ disconnects $M$.

The point sets considered in this paper all lie in a plane.

Definition of $F(C ; X, Y ; \epsilon)$. Let $C$ be any simple closed curve, $X$ and $Y$ distinct points of $C$, and $\epsilon$ any positive number. There exists a finite set of points $A_{1}, A_{2}, \cdots, A_{n},(n>2)$, such that (a) $A_{1}+A_{2}+\cdots+A_{n}$ contains $X+Y$, (b) $A_{1}, A_{2}, \cdots, A_{n}$ lie on $C$ in the order $A_{1} A_{2} \cdots A_{n} A_{1}$, and (c) $A_{i}$ and $A_{i+1}$ (subscripts are to be reduced modulo $n$ ) are the end points of an arc $t_{i}$ of diameter $<\epsilon$ which is a subset of $C$ not containing $A_{i+2}$. There exists a set of mutually exclusive arc segments $v_{1}, v_{2}, \cdots, v_{n}$ lying within $C$ such that $v_{i}+t_{i}$ is a simple closed curve $w_{i}$ of diameter $<\epsilon$. Let $J$ denote the simple closed curve

* See the discussion at the close of my paper, Transactions of this Society, vol. 33 (1931), p. 165.

$\dagger$ Presented to the Society, December 1, 1933.

$\ddagger$ Fundamenta Mathematicae, vol. 8 (1926), Problem 42, p. 376.

$\$$ B. Knaster, Sur un continu que tout sous-continu divise, Proceedings of the Polish Mathematical Congress, 1929, p. 59. 
$\sum_{1}^{n} A_{i}+v_{i}$. There exist $n$ infinite sequences of simple closed curves $C_{i j},(i=1,2, \cdots, n ; j=1,2, \cdots)$, such that (1) $C_{i j}$ contains $A_{i}$ but otherwise lies within $J$, (2) the sequence $C_{i 1}, C_{i 2}, C_{i 3}, \cdots$ has as sequential limit set the $\operatorname{arc} A_{i}+v_{i}+A_{i+1}$, (3) $C_{i j}$ is of diameter $<\epsilon,(4) C_{i j} \cdot C_{i k}=A_{i},(j \neq k)$, and $C_{i j} \cdot C_{h k}=0$, $(i \neq h)$, and (5) no point of $C_{i j}$ lies within any $C_{h k}$. The set $F(C ; X, Y ; \epsilon)$ is defined as the sum of all the curves $C_{i j}$ and the $n$ curves $w_{i}$ :

$$
F(C ; X, Y ; \epsilon)=\sum_{i=1}^{n}\left[w_{i}+\sum_{j=1}^{\infty} C_{i j}\right] .
$$

Definition of $M$. Let $E$ be any simple closed curve, $X$ and $Y$ any two points of $E$. Let $K_{1}$ denote a set $F(E ; X, Y ; 1)$. Then $K_{1}=\sum_{i=1}^{\infty} E_{1 i}$, where for each $i, E_{1 i}$ is a simple closed curve of diameter $<1$, and the common part of $E_{1 i}$ and the sum of the other curves $E_{11}, E_{12}, \cdots$ either is one point, or is two points. Thus $E_{1 i}$ contains distinct points $X_{1 i}$ and $Y_{1 i}$ such that no other point of $E_{1 i}$ belongs to $E_{1 j},(i \neq j)$. For each $i$ let $G_{1 i}$ be a set $F\left(E_{1 i} ; X_{1 i}, Y_{1 i} ; 1 / 2\right)$ and let $K_{2}$ be $G_{11}+G_{12}+\cdots$.

Suppose $K_{1}, K_{2}, \cdots, K_{n},(n>1)$, have been defined, $K_{1}$ being as defined above and, for each $i$, the following properties obtain:

I. $K_{i}$ is the sum of a countable set of simple closed curves $E_{11}, E_{12}, \cdots$.

II. Each curve $E_{i h}$ has, in common with the sum of the other curves $E_{i 1}, E_{i 2}, \cdots$, either one point or two points.

III. $X_{i h}$ and $Y_{i h}$ are distinct points of $E_{i h}$ such that no other point of $E_{i h}$ belongs to the sum of the other curves $E_{i 1}, E_{i 2}, \cdots$.

IV. No point is common to the interiors of two curves $E_{i n}$ and $E_{i k},(h \neq k)$.

V. $K_{i+1},(i<n)$, is a subset of the sum of $K_{i}$ and the interiors of all the curves $E_{i 1}, E_{i 2}, \cdots$.

VI. The subset of $K_{i+1},(i<n)$, which lies on and within $E_{i n}$ is a set $F\left(E_{i h} ; X_{i h}, Y_{i h} ; 1 /[i+1]\right)$.

For $n=2$, the sets $K_{1}$ and $K_{2}$ defined above have these properties. For each $i,(i \leqq n)$, let $U_{i}$ be the set of all points of $K_{i}$, each of which belongs to at least two curves of the set $E_{i 1}, E_{i 2}, \cdots$, and let $D_{i}$ denote $K_{i}$ plus the interiors of all the curves $E_{i 1}$, $E_{i 2}, \cdots$. 
For each $k$ let $G_{n k}$ be a set $F\left(E_{n k} ; X_{n k}, Y_{n k} ; 1 /[n+1]\right)$, and let $K_{n+1}$ be $G_{n 1}+G_{n 2}+\cdots$. Then it readily follows that the sequence $K_{1}, K_{2}, \cdots, K_{n}, K_{n+1}$ has the properties I-VI above. Hence there is an infinite sequence $K_{1}, K_{2}, \cdots$ with properties I-VI, $K_{1}$ being a set $F(E ; X, Y ; 1)$. Let $M$ be $K_{1}+K_{2}+\cdots$ plus all limit points. This is the same as the common part of $D_{1}, D_{2}, \cdots$.

Proof that $M-H$ is not Connected. Suppose $H$ is a nondegenerate proper subcontinuum of $M$. Suppose $M-H$ is connected. Now the components of $M-U_{n}$ are of diameter $<1 / n$. Hence there exists an $n$ such that $H$ contains a point $P$ of $U_{n}$. It will be shown that if $H$ contains a point of $U_{n}$, then it contains all of $U_{n}$. In view of this, and the fact that $U_{n}$ is a subset of $U_{n+1}$ and that $M=\left(U_{1}+U_{2}+\cdots\right)$ plus limit points, it follows that $H=M$, which is a contradiction.

It remains to show that if $H$ contains a point $P$ of $U_{n}$, then it contains all of $U_{n}$. Let $h$ be such that $P$ belongs to $E_{n h}$. The subset of $K_{n+1}$ which lies on and within $E_{n h}$ is a set $F\left(E_{n h} ; X_{n h}\right.$, $\left.Y_{n h} ; 1 /[n+1]\right)$. The points of $U_{n+1}$ in this set can be labeled $B_{1}, B_{2}, \cdots, B_{k}$, so that they lie on $E_{n h}$ in the order $B_{1} B_{2} \cdots B_{k} B_{1}$. Now each of the infinity of components of $K_{n+1}-B_{i}$ is a subset of a different component of $M-B_{i}$. Hence if $H$ contains $B_{i}$, and $M-H$ is connected, $H$ must contain all save one of these components. But $B_{i+1}$ is a limit point of the sum of the components of $K_{n+1}-B_{i}$. Hence, if $H$ contains $B_{i}$, it contains $B_{i+1}$. But for some $i, P=B_{i}$. Thus $H$ contains all the points of $U_{n+1}$ on $E_{n h}$, and therefore the one or two points of $U_{n}$ on $E_{n h}$. Now any two curves $E_{n h}$ and $E_{n k}$, of the set $E_{n 1}, E_{n 2}, \cdots$, can be joined by a finite chain $L_{1}, L_{2}, \cdots, L_{e}$ of curves of the set $E_{n 1}, E_{n 2}, \cdots, L_{1}$ having a point in common with $E_{n h}, L_{i}$ having a point in common with $L_{i+1},(i<e)$, and $L_{e}$ having a point in common with $E_{n k}$. Since these common points are in $U_{n}$, and $H$ contains a point of $U_{n}$ in $E_{n h}$, it readily follows, by repeated application of the above argument, that $H$ contains every point of $U_{n}$ in $E_{n h}+L_{1}+L_{2}+\cdots+L_{e}+E_{n k}$, and therefore $H$ contains every point of $U_{n}$.

DUKe UnIVERSITY 